\title{
Pregnancy intentions among female sex workers: recognising their rights and wants as mothers
}

\author{
Putu Duff, ${ }^{1}$ Jeannie Shoveller, ${ }^{2}$ Cindy Feng, ${ }^{3}$ Gina Ogilvie, ${ }^{4}$ \\ Julio Montaner, ${ }^{5}$ Kate Shannon ${ }^{6}$
}

- Additional material is published online only. To view please visit the journal online (http://dx.doi.org/10.1136/jprhc2012-100532)

For numbered affiliations see end of article.

\section{Correspondence to \\ Dr Kate Shannon, Department of Medicine, University of British Columbia, Gender and Sexual Health Initiative, BC Centre for Excellence in HIV/AIDS, St. Paul's Hospital, 608-1081 Burrard Street, Vancouver, British Columbia, Canada V6Z 1Y6; gshi@cfenet.ubc.ca}

Received 30 October 2012 Revised 6 January 2014 Accepted 23 June 2014 Published Online First 23 July 2014

\section{CrossMark}

\footnotetext{
To cite: Duff P, Shoveller J, Feng C, et al. J Fam Plann Reprod Health Care 2015:41:102-108.
}

\section{ABSTRACT}

Objective To better understand the prevalence and correlates of pregnancy intentions among female sex workers (FSWs).

Design Cross-sectional analysis using data from an open prospective cohort of street and offstreet FSWs in Vancouver, Canada, in partnership with local sex work and community agencies.

Methods FSWs were recruited through outreach to street and off-street locations (e.g. massage parlours, micro-brothels) and completed interviewer-administered questionnaires and HIV/ sexually transmitted infection testing. Bivariable and multivariable logistic regression was used to evaluate correlates of pregnancy intention, based on a 'yes' or 'no' response to the question "Are you planning on have any (any more) children in the future?".

Results Of the 510 women, 394 (77.3\%) reported prior pregnancy, with $140(27.5 \%)$ of the entire sample reporting positive pregnancy intentions. Regarding ethnicity, 35.3\% were Caucasian and $26.3 \%$ were Asian/visible minority, with no differences in pregnancy intention by ethnicity or HIV status; $38.4 \%$ reported Canadian Aboriginal ancestry. In our final multivariable model, servicing clients in formal indoor settings, inconsistent condom use by clients, younger age, and intimate partner violence (IPV) were associated with pregnancy intention.

Conclusions FSWs may have pregnancy intention levels similar to that of women in other occupations. Policy changes are needed to improve FSWs' access to integrated HIV and reproductive health services and harm reduction services, particularly for FSWs experiencing IPV.

\section{INTRODUCTION}

Though risks for HIV and pregnancy are intimately intertwined, research on female sex workers (FSWs) has focused almost exclusively on disease prevention

\section{Key message points}

- Female sex workers (FSWs) have pregnancy intentions similar to women in other professions, highlighting a need to better understand and attend to FSWs' reproductive needs.

- Improved access to sexual and reproductive health (SRH) services is needed for FSWs working in hidden formal indoor establishments, to better support their pregnancy intentions.

- Legal frameworks are required that better support FSWs' reproductive needs, alongside integrated SRH services that are focused on harm reduction and which address intimate partner violence.

and transmission, while overlooking the reproductive health (RH) needs and wants of this population. ${ }^{1}$ FSWs have been negatively portrayed in the media, by the public, and often by public health providers as 'vectors of disease', immoral women and/or criminals, and they are rarely viewed in their role as parents, perhaps because they do not conform to society's perception of 'good mothers'. ${ }^{2}$ Diverted research and practice attention from the reproductive rights and needs of female FSWs also may contribute to the high level of unmet reproductive need among this population. ${ }^{3}$ Understanding the pregnancy intentions of FSWs is necessary to inform comprehensive public health programmes that can meet their reproductive needs and desires.

Despite emerging evidence of high levels of pregnancy and parenting among FSWs, ${ }^{4} 5$ little is known about the 
pregnancy intentions of this population, particularly among FSWs in industrialised settings such as in North America. The few empirical studies that have explored FSWs' RH and pregnancy patterns were conducted among non-drug using FSWs in nonindustrialised countries, and did not explicitly investigate pregnancy intentions. ${ }^{6} 7$ Instead, these studies documented levels of abortions as a proxy measure of unintended pregnancy, which probably underestimates the true rate of unintended pregnancy by not accounting for unintended pregnancies resulting in live births or those that ended in spontaneous abortion. These and most other existing studies assessed pregnancy intention retrospectively (i.e. pregnancy intentions before vs after conception, or using unintended pregnancy as a proxy for pregnancy intention), ${ }^{8}$ and did not have the capacity to capture true levels of pregnancy intentions.

While we identified no studies examining pregnancy intentions among FSWs, pregnancy intention studies among marginalised populations and women living with HIV may contribute to our understanding of pregnancy intentions among FSWs, given high rates of HIV (23\% among street-based FSWs) and marginalisation among this population. ${ }^{9}$ A recent study among HIV-positive women of reproductive age living in Vancouver, Canada revealed pregnancy intention levels approaching that of the general population, with age, marital status and ethnicity being significant predictors of wanting to become pregnant. ${ }^{10}$ In other studies, pregnancy intentions of persons living with HIV/AIDS have been shown to vary according to a variety of other individual and social factors, including parity, social expectations and cultural norms, ${ }^{11}$ and knowledge of mother-to-child-transmission of HIV. ${ }^{12}$ Social networks, such as immediate and extended family members and friends, also significantly impact women's pregnancy desires and intentions through approval or encouragement of childbearing, ${ }^{13}$ including the intentions of intimate partners. ${ }^{14}$

The enforcement of Canada's sex work laws has been posited to fuel stigma (including stigma towards parenting FSWs), ${ }^{15-17}$ and to reduce access to health services, ${ }^{18}$ which may deter FSWs from wanting to start a family. However, the legal landscape of sex work in Canada is changing, with a recent (December 2013) Supreme Court of Canada decision striking down three criminal sanctions targeting sex work that had harmful consequences on FSW' health and safety. ${ }^{19} 20$

There is limited literature regarding the pregnancy intentions of marginalised women. ${ }^{21}$ We found no empirical studies measuring correlates of prospective pregnancy intentions among FSWs. This study therefore sought to examine the correlates of pregnancy intention among a cohort of FSWs in Vancouver, Canada. Gaining an understanding of the pregnancy intentions of FSWs is an important first step in identifying their RH needs and in developing recommendations for policy and programming that will improve RH rights, access and outcomes for FSWs in Vancouver and elsewhere. ${ }^{22}$

\section{METHODS}

\section{Study design}

Sampling

These analyses drew on cross-sectional data from an open prospective cohort of street and off-street FSWs in Vancouver, in partnership with local sex work and community agencies. The sample used AESHA (An Evaluation of Sex Workers' Health Access) baseline data collected between January 2010 and October 2011. As in a previously reported study, ${ }^{23}$ eligibility criteria included being female (inclusive of transgender) and having exchanged sex for money within the past month. As in our previous studies, FSWs were recruited using time-location sampling both at outdoor venues (e.g. streets, alleys) and indoor sex work venues (e.g. massage parlours, micro-brothels and FSWs' homes) through daytime and late-night outreach. Online recruitment targeting online solicitation spaces was also used. Time-space sampling is a strategy that recruits hard-to-reach populations by sampling in places and times where they often congregate, and uses physical spaces, rather than persons, as the primary sampling unit. ${ }^{24}$ As previously, community mapping with former/current FSWs was employed to locate sex work venues and spaces or 'strolls'.

\section{Instrument}

Participants were required to provide informed consent prior to completing an intervieweradministered questionnaire. The questionnaire elicited a wide range of information, described in detail below. In addition, a project nurse administered a brief health-related questionnaire that elicited information on the broader sexual, reproductive and physical health needs of women, including pregnancy history, pregnancy intentions, contraceptive usage and health services access. Following the pre-test counselling, blood was drawn for syphilis, HSV-2 antibody and hepatitis $\mathrm{C}$ virus testing. HIV was screened for using INSTI $^{\mathrm{TM}}$ (Biolytical Laboratories, Richmond, BC, Canada) rapid tests (followed by Western blots to confirm positive tests). Urine samples were also collected to test for gonorrhoea and chlamydia. Participants were compensated for their time and expertise with Canadian $\$ 40$ at baseline and at each follow-up visit for their time and expertise. The study received ethical approval from University of British Columbia's Research Ethics Board.

\section{Dependent variable}

The dependent variable, pregnancy intention, was defined as a response of "yes trying to become pregnant now" or "yes in the future" or "yes, currently 
pregnant" vs "no" to the following survey item: "Are you planning/hoping to have any (more) children in the future?”

\section{Independent variables}

Independent variables were considered based on $a$ priori knowledge from the literature as well as other hypothesised confounders. Age was measured as a continuous variable, and ethnicity was defined as Caucasian, Canadian Aboriginal/indigenous ancestry (inclusive of First Nations, Métis, Inuit and non-status First Nations) or Asian/visible minority (Chinese, Vietnamese, Thai, Filipina, Indian, Pakistani, Bangladeshi, Black, Latina). Other socio-demographic factors considered included migrant status (not born in Canada vs born in Canada) and educational status (high school graduate vs less than high school education). Individual drug use patterns of interest were: past 6 months use of injection and non-injection drugs (excluding marijuana and alcohol). In the light of the pregnancy intentions literature, we also accounted for interpersonal factors such as having had a male intimate partner (non-commercial) within the previous 6 months and inconsistent condom use by clients and intimate partners. ${ }^{12}$

Given the documented association between intimate partner violence (IPV) and unintended pregnancy, ${ }^{25-27}$ we used the World Health Organization standardised scale for IPV (V.9) that captures any or all of physical, sexual and emotional violence perpetrated by a primary male intimate/sexual partner in the past 6 months. ${ }^{28}$ Client-perpetrated physical and/or sexual violence within the past 6 months was also included in our analysis. Since pregnancy history and parity have also been documented as predictors of pregnancy desires/ intention, ${ }^{11}$ we accounted for number of pregnancies, number of children in the mother's custody, whether a child had ever been removed by child welfare services, and any barriers to pregnancy and mothering services (yes vs no) in our analysis.

Finally, since we hypothesised that contraceptive usage [inconsistent condom use by clients, and use of hormonal contraceptives (birth control pills, injectable hormones)] or work environmental factors of interest such as place of servicing clients, could influence pregnancy intentions, these variables were also included in our analysis. Primary place of servicing clients was categorised as street/public spaces; informal indoor spaces (e.g. bars, saunas, hourly rental rooms); and formal sex work establishments (e.g. brothels/quasi brothels, massage parlours, beauty establishments).

\section{Statistical analyses}

The analytic sample was restricted to those women who provided a valid response to the pregnancy intentions question, resulting in a final sample size of 510 participants. Initially, we examined bivariate associations between individual, drug-related, interpersonal and environmental variables with pregnancy intentions. For these comparisons, we employed Pearson's chi-square $\left(\chi^{2}\right)$ test for analysis of dichotomous, categorical variables and Wilcoxon rank sum test for continuous variables. Logistic regression was used to generate odds ratios (ORs) with 95\% confidence intervals $(95 \% \mathrm{CI})$ generated for all categorical dichotomous variables. When observations were less than or equal to five, Fisher's exact test of probability was used to generate $p$ values. Variables with $p$ values $<0.20$ were included in the multivariable logistic regression model and Akaike Information Criteria (AIC) selection was employed to arrive at the final multivariable model.

The final model was tested for multi-collinearity. Two variables (having a male intimate partner and inconsistent condom use with an intimate partner) were removed from the model due to collinearity with (physical, emotional or sexual) IPV in the last 6 months. Given the significant association between the IPV variable and both 'having a male intimate partner' and 'inconsistent condom use with an intimate partner', as well as literature suggesting that IPV is high among pregnant and parenting women, ${ }^{25-27} \mathrm{IPV}$ was therefore considered the more important variable to include. The adjusted odds ratios (AORs) and $p$ values were calculated for variables remaining in the final model after AIC selection (displayed in online Supplementary Table S2). As in previous studies, ${ }^{29}$ variables that retained significance at the $p<0.05$ level in the multivariable model after adjusting for potential confounders were considered statistically significant. Variables with $p$ values $<0.10$ in our final multivariable model were considered marginally significant, as in previous studies. ${ }^{30-32}$

\section{RESULTS}

Of 510 women, 140 (27.5\%) expressed an intention to have (more) children. Sample characteristics are presented in online Supplementary Table S1, stratified by pregnancy intention. The median age for women expressing pregnancy intention was 29 [interquartile range (IQR) 25-35], compared to a median of 38 (IQR 31-44) among those not desiring children. There were no differences in pregnancy intentions by ethnicity, with 49 (34.0\%) women with Aboriginal ancestry, 50 (34.9\%) Caucasian and 44 (30.8\%) Asian women (primarily migrant/new immigrant workers) indicating wanting (more) children. Approximately half $(49.8 \%)$ of our sample had a high school education, and 32\% reported homelessness in the past 6 months.

Online Supplementary Table S2 displays bivariate and multivariable associations with pregnancy intentions. In multivariable analysis, inconsistent condom use by clients (AOR $=1.95 ; 95 \%$ CI 1.11-3.43; $p=0.002)$, servicing clients in formal indoor spaces vs public outdoor spaces $\quad(\mathrm{AOR}=1.95 ; 95 \%$ CI 1.17-3.26; 
$p=0.011$ ) were associated with elevated odds of wanting children after controlling for potential confounders. IPV in the last 6 months (physical, sexual or emotional) was associated with a $64 \%$ increased odds of pregnancy intentions $(\mathrm{AOR}=1.64 ; 95 \%$ CI $0.97-$ 2.78 ), although this association was only marginally significant $(p=0.065)$. Younger age, measured as a continuous variable $(\mathrm{AOR}=0.89 ; 95 \%$ CI $0.87-0.92$; $p=<0.001$ ), was related to positive pregnancy intentions, after adjusting for potential confounders.

\section{DISCUSSION}

To our knowledge, this study is the first to document the pregnancy intentions of FSWs. Approximately one in four $(27.5 \%)$ of the women in our study expressed an intention to become pregnant, with younger age, inconsistent condom use by clients, IPV in the past 6 months, and servicing clients in indoor settings being significantly associated with increased pregnancy intentions after adjusting for potential confounders. The pregnancy intention level among our sample appears to be lower than that of the general female Canadian population $(37.5 \%),{ }^{11}$ and marginally higher compared to reported rates among HIV-positive women in our setting (25\%), ${ }^{33}$ although more work is needed to fully understand how FSWs' pregnancy intentions compare to women of other occupations.

These findings challenge stereotypes about FSWs (e.g. being unlikely to want to be mothers) and support qualitative accounts that FSWs do desire pregnancy, just as women in other occupations do. Qualitative research describes that women place high importance on children and on their role as mothers, and view motherhood as fulfilling their need to give and receive love. ${ }^{11}$ Some accounts portray motherhood as giving FSWs' lives meaning and structure, and this may often be the only source of life satisfaction. $^{34}$ Though the level of pregnancy intention among FSWs in our sample is slightly lower than that of the general population, these findings highlight a need to better understand the reproductive needs and desires of this population.

In the light of recent findings that indicate condoms to be FSWs' primary method of birth control, ${ }^{34}$ in the absence of access to other forms of birth control it was not unexpected that inconsistent condom use was associated with elevated odds of pregnancy intention in this study. Our results show that women who want children have $95 \%$ increased odds of inconsistent condom use with commercial sex clients, suggesting a potential increased HIV/sexually transmitted infection (STI) risk pathway. These results suggest an urgent need for integrated HIV and RH services that balance the need for HIV prevention with sensitivity to FSWs' reproductive rights and needs. Such services should be non-judgmental, supporting the use of barrier contraceptives (e.g. male or female condoms) for women not desiring pregnancy, whilst also providing information and support for achieving safe pregnancies for FSWs wanting children. They should include improved access to antiretroviral and addictions treatment, STI screening and treatment, perinatal services (including prevention of mother-to-child transmission services), family planning information, and fertility treatment.

Of concern, our results show a $64 \%$ increased odds of IPV among FSWs who intend to have (more) children. While there is a substantial body of work linking domestic violence and pregnancy in the general population, this research has almost exclusively focused on pregnancy and the early postpartum periods. ${ }^{25} 35 \quad 36$ Instead, our results suggest that women experiencing domestic abuse are more likely than those not experiencing abuse or without a stable partner to report pregnancy intentions. The mechanism linking IPV and positive pregnancy intention is unclear and warrants further investigation. Given that previous literature has shown higher rates of IPV among pregnant and parenting women, our finding may be reflective of a climate of IPV within relationships where women either already have children or are actively discussing having children. Another possible explanation for our finding is that FSWs experiencing IPV may desire the safer, more stable family life that having a child might be presumed to offer. Further qualitative research, and in-depth work on pregnancy and violence experiences, is recommended. Of importance, this finding draws attention to the often overlooked but prevailing normalisation and 'everyday' violence experienced by FSWs, both in their private and work lives.

Previous qualitative studies among street-based FSWs have suggested high rates of various forms of violence from clients, pimps and intimate partners. ${ }^{37} 38$ The culture of violence towards FSWs may in part stem from the highly criminalised and stigmatised nature of sex work and poverty, and as such, community-level interventions to reduce poverty and stigma surrounding sex work could be beneficial.

On a programming level, there is a need for harm reduction services for FSWs, including trauma counselling and respite from abusive relationships. In particular, there is a need for structural and gender-transformative interventions that target male gender inequity norms among male perpetrators of violence. ${ }^{39}$

Of particular interest, FSWs who serviced clients in formal sex work establishments had almost two-fold increased odds of wanting to have children compared to those that serviced clients in outdoor/or public spaces or informal indoor spaces. A possible explanation is that given the highly criminalised and stigmatised context of sex work in Canada, FSWs may feel more able to keep their private and personal lives separate while working in indoor sex work establishments. Formal indoor sex work establishments may also 
provide a safer, more stable environment that may better support women's intentions to have children. This hypothesis is supported by qualitative research that suggests that maintaining secrecy about FSWs' work from friends, family and their children is a priority for parenting FSWs. ${ }^{34}$ In addition to potentially offering a form of privacy, formal indoor work environments may offer greater and/or more stable financial remuneration compared to outdoor sex work or other informal sex work arrangements, ${ }^{40}$ as well as the potential to access social support networks within the work environment, ${ }^{40}$ which may affect FSWs' pregnancy intentions.

Given the recent changes to Canada's criminal code, these findings highlight a need to pursue a framework that promotes FSWs' health and safety, and improves access to formal indoor establishments that may better support their reproductive right to have children. A decriminalisation framework may offer these benefits. This has had positive impacts on FSWs' health and safety in New Zealand since its inception in 2003, including reduced violence and improved access to health care and support services. ${ }^{41}$

Our finding of greater pregnancy intention among younger women is consistent with the literature, ${ }^{10}$ and may be explained by lower parity and unfulfilled pregnancy desires among younger women. Younger FSWs in our setting have been shown to have increased health risks due to elevated levels of homelessness, ${ }^{104243}$ and avoidance of health and support services, which may have serious consequences on the RH of FSWs and their children.

Together, these findings highlight a critical need for accessible and targeted $\mathrm{RH}$ services that support FSWs' reproductive choice, from pregnancy prevention (e.g. contraception, abortion services) through to pregnancy and child rearing (e.g. family planning, perinatal care, parental support services). Given the close ties between HIV and pregnancy, innovative service delivery models that integrate HIV and $\mathrm{RH}$ services (e.g. HIV integrated into RH services or vice versa) may hold promise.

Additionally, these findings concur with previous studies that suggest that the geographical location and method of service delivery are paramount to promoting health care access among FSWs. ${ }^{44}$ In particular, integrated services within close proximity to FSWs' workplaces or homes, or outreach to FSWs' workplaces, ${ }^{45}$ may provide immense benefit in mitigating existing elevated levels of harms faced by them and help interrupt a pattern of accumulating damage over their life course and that of their children. Examples of accessible, innovative models include the Esselen Street Clinic in Johannesburg, South Africa that provides (on-site and mobile) integrated RH/HIV services, ${ }^{46}$ and the Sheway project in Vancouver that offers woman-centred, harm-reduction treatment services, and parental support to mothers and their families. ${ }^{47}$
This study has a number of limitations. The clandestine nature of sex work makes identifying a sampling frame, and randomly selecting participants that are representative of the population, a challenge. To address this limitation, time-space sampling and social mapping were used that systematically sample women at times and locations where they often congregate. ${ }^{10}$ Due to the sensitive nature of the topic, social desirability bias cannot be excluded and may have resulted in underreporting of pregnancy intention. Using a dichotomous measure of pregnancy intention (i.e. intending to become pregnant vs not intending to become pregnant) may not capture ambivalence with regard to pregnancy intentions, which has been associated with higher numbers of pregnancies compared to women who definitely did not want children in lower-middle income countries (e.g. Morocco, Malaysia). ${ }^{48} 49$ Finally, given that our study findings are specific to FSWs in an urban North American setting, our study findings may not be generalisable to FSWs in other settings, particularly those with differing policies and outlook on sex work.

\section{CONCLUSIONS AND FUTURE DIRECTIONS}

These findings suggest that FSWs may have pregnancy intentions similar to women in other occupations, challenging widely held assumptions. They indicate a need for integrated HIV, RH and harm reduction services that cater to the needs of women experiencing IPV and inconsistent condom use with clients. Such services should support FSWs' choices (i.e. provide contraception, termination, pre- and postnatal care and childrearing supports), offer (or link to) counselling and respite from abusive relationships, and be delivered at or near FSWs' workplaces. Alternative legal frameworks such as the decriminalisation of sex work might further improve FSWs' reproductive rights to have children by increasing access to formal indoor sex work establishments and encouraging the delivery of targeted, integrated services at or near these locations.

\section{Author affiliations}

${ }^{1}$ Research Assistant and PhD Candidate, British Columbia Centre for Excellence in HIV/AIDS, St Paul's Hospital, Vancouver and School of Population and Public Health, University of British Columbia, Vancouver, British Columbia, Canada

${ }^{2}$ Professor, School of Population and Public Health, University of British Columbia, Vancouver, British Columbia, Canada

${ }^{3}$ Assistant Professor, School of Public Health, University of Saskatchewan, Saskatoon, Saskatchewan, Canada

${ }^{4}$ Associate Director of the Division of STI/HIV Prevention, British Columbia Centre for Disease Control, Vancouver, British Columbia, Canada

${ }^{5}$ Director, British Columbia Centre for Excellence in HIV/AIDS, St Paul's Hospital, Vancouver and 
Department of Medicine, University of British Columbia, St Paul's Hospital, Vancouver, British Columbia, Canada

${ }^{6}$ Associate Professor and Director of the Gender and Sexual Health Initiative, British Columbia Centre for Excellence in HIV/AIDS, St Paul's Hospital, Vancouver; School of Population and Public Health, University of British Columbia, Vancouver; and Department of Medicine, University of British Columbia, St Paul's Hospital, Vancouver, British Columbia, Canada

Acknowledgements The authors thank all those who contributed their time and expertise to this project, including participants, partner agencies and the AESHA Community Advisory Board. They wish to acknowledge Peter Vann, Calvin Lai, Eric Fu, Ofer Amram, Jill Chettiar, Alex Scot and Kathleen Deering for their research and administrative support.

Funding This research was supported by operating grants from the US National Institutes of Health (R01DA028648) and Canadian Institutes of Health Research (HHP-98835).

Competing interests Putu Duff is supported by PHIRNET: The Population Health Intervention Research Network, which is funded by the Canadian Institutes for Health Research) and UBC's Liu Institute for Global Issues. Kate Shannon is supported by US National Institutes of Health and the Canadian Institutes of Health Research (R01DA028648). Jeannie Shoveller is supported by an Applied Public Health Chair from the Canadian Institutes of Health Research and a Senior Scholar Award from the Michael Smith Foundation for Health Research.

Ethics approval University of British Columbia/Providence Health Care Research Ethics Board.

Provenance and peer review Not commissioned; externally peer reviewed.

\section{REFERENCES}

1 Basu A, Dutta MJ. "We are mothers first": localocentric articulation of sex worker identity as a key in HIV/AIDS communication. Women Health 2011;51:106-123.

2 McClelland GT, Newell R. A qualitative study of the experiences of mothers involved in street-based prostitution and problematic substance use. J Res Nurs 2008;13:437-447.

3 Chacham AS, Diniz SG, Maia MB, et al. Sexual and reproductive health needs of sex workers: two feminist projects in Brazil. Reprod Health Matters 2007;15:108-118.

4 Feldblum PJ, Nasution MD, Hoke TH, et al. Pregnancy among sex workers participating in a condom intervention trial highlights the need for dual protection. Contraception 2007;76:105-110.

5 Delvaux T, Crabbe F, Seng S, et al. The need for family planning and safe abortion services among women sex workers seeking STI care in Cambodia. Reprod Health Matters 2003;11:88-95.

6 Bautista CT, Mejia A, Leal L, et al. Prevalence of lifetime abortion and methods of contraception among female sex workers in Bogota, Colombia. Contraception 2008;77:209-213.

7 Elmore-Meegan M, Conroy RM, Agala CB. Sex workers in Kenya, numbers of clients and associated risks: an exploratory survey. Reprod Health Matters 2004;12:50-57.

8 Kavanaugh ML, Schwarz EB. Prospective assessment of pregnancy intentions using a single- versus a multi-item measure. Perspect Sex Reprod Health 2009;41:238-243.
9 Shannon K, Bright V, Duddy J, et al. Access and utilization of HIV treatment and services among women sex workers in Vancouver's Downtown Eastside. J Urban Health 2005;82:488-497.

10 Ogilvie GS, Palepu A, Remple VP, et al. Fertility intentions of women of reproductive age living with HIV in British Columbia, Canada. AIDS 2007;21(Suppl. 1):S83-S88.

11 Loutfy MR, Hart TA, Mohammed SS, et al. Fertility desires and intentions of HIV-positive women of reproductive age in Ontario, Canada: a cross-sectional study. PLOS ONE 2009;4:e7925.

12 Nattabi B, Li J, Thompson SC, et al. A systematic review of factors influencing fertility desires and intentions among people living with HIV/AIDS: implications for policy and service delivery. AIDS Behav 2009;13:949-968.

13 Miller WB. Childbearing motivations, desires, and intentions: a theoretical framework. Genet Soc Gen Psychol Monogr 1994;120:223-258.

14 Finocchario-Kessler S, Sweat MD, Dariotis JK, et al. Childbearing motivations, pregnancy desires, and perceived partner response to a pregnancy among urban female youth: does HIV-infection status make a difference? AIDS Care 2012;24:1-11.

15 Kurtz SP, Surratt HL, Kiley MC, et al. Barriers to health and social services for street-based sex workers. J Health Care Poor Underserved 2005; 16:345-361.

16 Lazarus L, Deering KN, Nabess R, et al. Occupational stigma as a primary barrier to health care for street-based sex workers in Canada. Cult Health Sex 2012;14:139-150.

17 Sloss CM, Harper GW, Budd KS. Street sex work and mothering. Journal of the Association for Research on Mothering 2005;6:102-115.

18 Shannon K, Rusch M, Shoveller J, et al. Mapping violence and policing as an environmental-structural barrier to health service and syringe availability among substance-using women in street-level sex work. Int J Drug Policy 2008;19:140-147.

19 Shannon K, Strathdee SA, Shoveller J, et al. Structural and environmental barriers to condom use negotiation with clients among female sex workers : implications for HIV-prevention strategies and policy. Am J Public Health 2009;99:659-665.

20 Shannon K, Kerr T, Strathdee SA, et al. Prevalence and structural correlates of gender-based violence among a prospective cohort of street-based sex workers. BMJ 2009;339:b2939.

21 Weber AE, Tyndall MW, Spittal PM, et al. High pregnancy rates and reproductive health indicators among female injection-drug users in Vancouver, Canada. Eur J Contracept Reprod Health Care 2003;8:52-58.

22 Overs C, Hawkins K. Can rights stop the wrongs? Exploring the connections between framings of sex workers' rights and sexual and reproductive health. BMC Int Health Hum Rights 2011;11(Suppl. 3):S6.

23 Shannon K, Bright V, Allinott S, et al. Community-based HIV prevention research among substance-using women in survival sex work: the Maka Project Partnership. Harm Reduct J 2007;4:20.

24 Stueve A, O’Donnell LN, Duran R, et al. Time-space sampling in minority communities: results with young Latino men who have sex with men. Am J Public Health 2001;91:922-926.

25 Campbell JC. Health consequences of intimate partner violence. Lancet 2002;359:1331-1336.

26 Kelly PJ, Ramaswamy M. The association between unintended pregnancy and violence among incarcerated men and women. J Community Health Nurs 2012;29:202-213. 
